\title{
Laboreal
}

Volume $12 \mathrm{~N}^{\circ} 1$ | 2016

Os Equipamentos de Proteção Individual (EPI):

protetores, mas nem sempre

\section{Los guantes antivibratorios: ¿une avenida de prevención eficaz y aplicable?}

As luvas antivibráteis: uma via de prevenção eficaz e aplicável?

Les gants antivibratiles: une avenue de prévention efficace et applicable?

Anti-vibration gloves: an option of effective and applicable prevention?

\section{Alice Turcot y Michel Lehoux}

\section{(2) OpenEdition}

\section{Journals}

Edición electrónica

URL: http://journals.openedition.org/laboreal/3157

DOI: $10.4000 /$ laboreal.3157

ISSN: 1646-5237

Editor

Universidade do Porto

Referencia electrónica

Alice Turcot y Michel Lehoux, «Los guantes antivibratorios: ¿une avenida de prevención eficaz y aplicable? », Laboreal [En línea], Volume 12 N$^{0} 1$ | 2016, Publicado el 01 julio 2016, consultado el 08 octubre 2019. URL : http://journals.openedition.org/laboreal/3157 ; DOI : 10.4000/laboreal.3157

Este documento fue generado automáticamente el 8 octubre 2019.

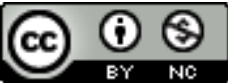

Laboreal está licenciado com uma Licença Creative Commons - Atribuição-NãoComercial 4.0 Internacional. 


\section{Los guantes antivibratorios: ¿une avenida de prevención eficaz y aplicable?}

As luvas antivibráteis: uma via de prevenção eficaz e aplicável?

Les gants antivibratiles: une avenue de prévention efficace et applicable?

Anti-vibration gloves: an option of effective and applicable prevention?

Alice Turcot y Michel Lehoux

\section{NOTA DEL EDITOR}

http://dx.doi.org/10.15667/laborealxii0116at

Manuscrito recebido em: março/2016

Aceite após peritagem: junho/2016

Se agradece al señor Pierre Marcotte, Instituto de investigación Robert-Sauvé en salud y seguridad laboral.

\section{INTRODUCCIÓN}

1 La exposición prolongada a las vibraciones que producen las herramientas vibrantes está asociada al síndrome vibratorio que presentan los trabajadores expuestos a estas vibraciones (Griffin \& Bovenzi, 2002). Se pueden establecer diferentes estrategias de prevención en el lugar de trabajo a fin de disminuir o suprimir la exposición de los trabajadores a las vibraciones de manos y brazos. Entre las estrategias posibles, se encuentra la reducción de las vibraciones a partir del origen de estas mismas, mediante el uso de herramientas que vibren menos, adaptadas para el trabajo y bien mantenidas, la aislación del operador de la herramienta o de la máquina ya sea mediante mangos antivibratorios o mediante el uso de guantes antivibratorios, el mejoramiento de la 
ergonomía como el control de las posturas incómodas o que limitan los esfuerzos musculares y, finalmente, el mejoramiento de la organización del trabajo gracias a la reducción del tiempo de exposición o incluso a la rotación de los puestos de trabajo (Health and Safety Executive, 1997; Marcotte, 2011).

¿Qué se sabe de la eficacia de los guantes antivibratorios respecto a la reducción de la transmisión de las vibraciones de la herramienta hacia el sistema mano-brazo o incluso para prevenir la enfermedad? ¿Qué factores son favorables o constituyen un obstáculo en el uso de los guantes? ¿La disponibilidad de los guantes antivibratorios en el lugar de trabajo favorece su utilización? Intentaremos responder a estas preguntas a partir de la revisión de la literatura y de un análisis cualitativo realizado con los trabajadores.

\section{MÉTODO}

\subsection{Revisión de la literatura}

3 Una búsqueda en PubMed y EBSCOhost, en el período comprendido entre 1970 y 2014, con las palabras clave manos y brazos, síndrome de vibración (hand-arm vibration syndrome) y guantes antivibratorios, nos permitió encontrar 62 artículos. Después de haber eliminado los artículos repetidos y los que no eran pertinentes, se analizaron 47 artículos en inglés y en francés y 2 resúmenes de artículos en lengua extranjera. Se analizaron documentos $(n=5)$ de organismos internacionales como Health and Safety Executive, el Instituto nacional de investigación y seguridad de Francia y el Instituto für Arbeitsschutz (IFA). También se incluyeron en el análisis artículos provenientes de seminarios $(\mathrm{n}=2)$ o de conferencias sobre el síndrome vibratorio $(\mathrm{n}=11)$. Finalmente, se analizaron estudios relativos al confort de los guantes y a la destreza manual. Se analizaron 72 textos y artículos en total.

\subsection{Estudio de campo}

4 Se invitó a treinta (30) trabajadores a participar al estudio utilizando dos tipos de guantes antivibratorios durante una semana cada uno. Según el fabricante, estos guantes contienen burbujas de aire, lo que los convierte en antivibratorios. El modelo A cubre los dedos hasta la mitad, asegura la muñeca y está hecho de nailon en la parte superior y de cuero en la parte de la palma de la mano. El modelo B cubre los dedos por completo y es de cuero (Figura 1). 
Figura 1: Tipos de guantes puestos a prueba por los trabajadores

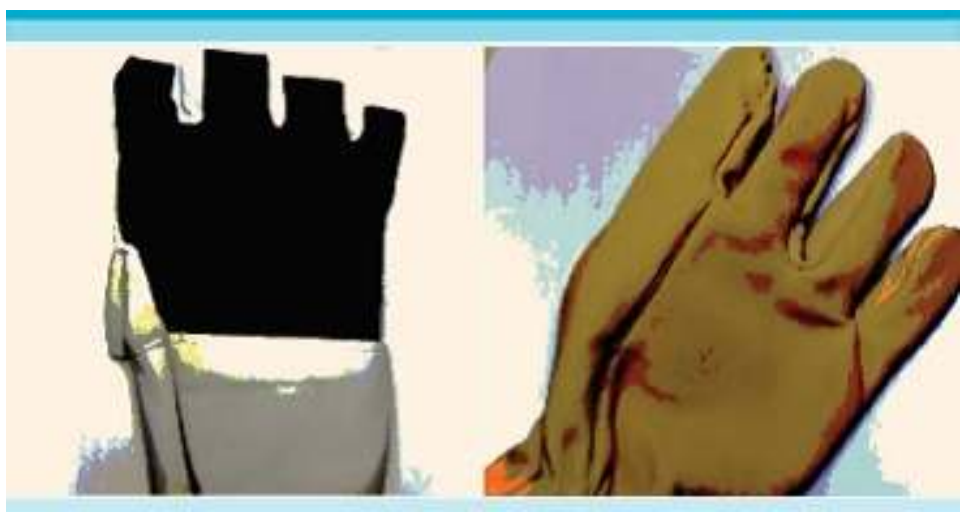

5 El fabricante de estos dos tipos de guantes dice que cumplen con la norma ISO 10819:1996 (Organisation international de normalisation, 1996). Sin embargo, el guante A no puede considerarse como antivibratorio por la norma ISO 10819, puesto que no cubre los dedos por completo.

Los trabajadores ocupan puestos en seis empresas en los siguientes sectores: fabricación de duchas y baños de material compuesto, fundición, fabricación de muebles, corte de madera o fabricación de productos de metal. Realizan trabajos de amolado, arenado, corte, pulido o corte de madera.

7 Se distribuyó el primer cuestionario de evaluación de manera autoadministrada. Trataba sobre el estado de salud del sistema mano-brazo, así como sobre el tipo de herramientas vibrantes utilizadas en el trabajo. El segundo cuestionario autoadministrado trataba sobre cómo los trabajadores percibían la eficacia de los guantes para atenuar las vibraciones, el confort, el nivel de destreza manual, el confort térmico, la agilidad, el ajuste a la mano, la adherencia y las incomodidades o los problemas ocasionados al usarlos.

8 De los 30 trabajadores, 12 aceptaron pasar una entrevista individual al final del período de prueba. El objetivo de dichas entrevistas consistía en obtener informaciones complementarias respecto del ensayo de los guantes, además de reunir las modificaciones que desearan aportar a los modelos de guantes.

9 Los trabajadores completaron un formulario de consentimiento para la participación en el estudio.

\section{REVISIÓN DE LA LITERATURA}

10 La literatura acerca de los guantes antivibratorios comprende cuatro ámbitos de estudio. Un primer ámbito trata sobre la medida de laboratorio respecto de la eficacia de los guantes para atenuar la transmisión de las vibraciones, según la norma ISO 10819 (1996/2013). El segundo ámbito hace referencia al perfeccionamiento de la metodología de esta norma. Un tercer ámbito trata sobre la eficacia de los guantes medida sobre el terreno, así como sobre los estudios experimentales o epidemiológicos respecto de la observación de efectos fisiológicos a nivel de la mano o respecto de la reducción del síndrome vibratorio. Finalmente, existen estudios sobre la interferencia del uso de los guantes en el desempeño laboral. La literatura se concentra especialmente en los dos primeros ámbitos. 


\subsection{Un poco de historia}

11 vibración transmitida al sistema mano-brazo (Christ, 1983; Goel \& Rim, 1987; Rens, Dubrulle \& Malchaire, 1987; Reynolds \& Jetzer, 1998). Estos primeros estudios recurrieron a montajes especiales, no normalizados, de acelerómetros ubicados a nivel de la mano y de los dedos. Dichos estudios exigían conocer el comportamiento del sistema mano-brazo sometido a estímulos vibratorios, así como la identificación de los parámetros que podrían afectar la respuesta del sistema mano-brazo. Recién en 1996 se establece una norma internacional ISO a fin de medir, en laboratorio, la atenuación de la transmisión de la vibración que procuran los guantes (ISO:10819, 1996).

En Japón, se estableció una norma similar en el 2007 (Shibata \& Maeda, 2008). En los Estados Unidos, se elaboró una norma equivalente en el 2002 y se la actualizó en el 2014 (American National Standards Institute, S3.40-2002/2014).

Los guantes antivibratorios forman parte de la categoría II de la norma europea EU Directive 89/686/EEC (Kaulbars \& Walther, 2012). Para pertenecer a dicha categoría, los guantes deben ser certificados por un organismo reconocido internacionalmente y tener la marca $\mathrm{CE}$, lo que indica que cumplen con la norma.

\subsection{Composición y funcionamiento de los guantes antivibratorios}

Los guantes antivibratorios están hechos con un material resiliente que cubre la palma y los dedos. Este material puede ser una membrana de elastómero, un material viscoelástico como el Gelfôm que se encuentra entre dos capas de espuma, una membrana constituida de dos capas de plástico de burbujas (air bladder) o incluso una membrana de alvéolos de aire unida a una bomba en miniatura. Estos últimos son eficaces cuando las celdillas de aire están infladas, lo que los hace más livianos que los de material viscoelástico. En general, el envoltorio externo del guante es de cuero o de un material recubierto de nitrilo (Boileau \& Boutin, 2003).

Los guantes antivibratorios actúan como almohadillas pasivas entre la mano y la superficie de la herramienta a fin de reducir la transmisión de la vibración al igual que el sistema de suspensión de un automóvil (Dong, et al., 2009). La almohadilla reduce la rigidez de la superficie de contacto entre la mano y el mango de la herramienta. La eficacia del guante para atenuar la vibración depende no sólo de las propiedades mecánicas del guante, sino también de la masa aparente del sistema mano-brazo. 
Figura 2: Medidas de la vibración según los ejes $x, y$ y $z$

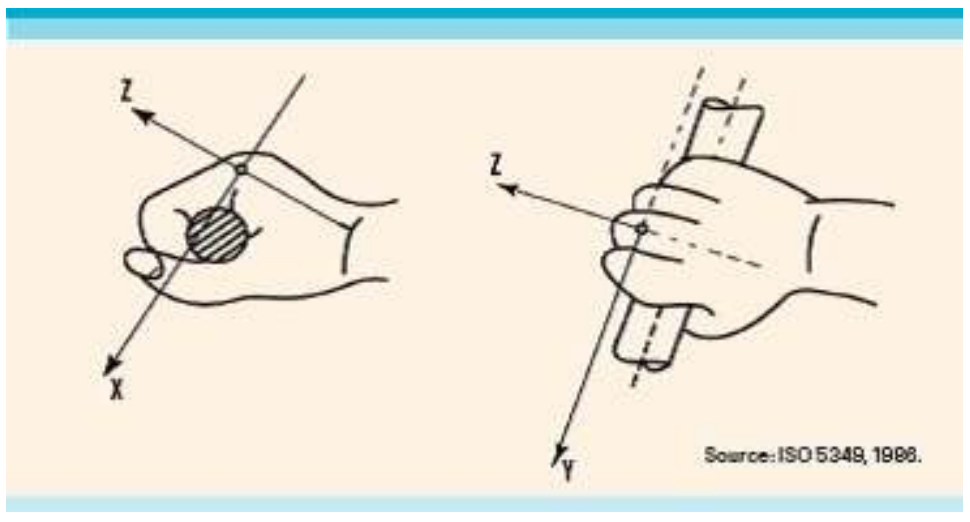

La eficacia del guante dependerá del mejoramiento del diseño del guante (Dong et al., 2009). La disminución de la rigidez y la optimización de la amortiguación aumentan la eficacia de la aislación de los guantes. La utilización de materiales más blandos o el aumento del espesor del material a nivel de la palma de la mano permite obtener dicha eficacia (Dong et al., 2009). En consecuencia, esto acarrea inconvenientes mayores respecto del control de la herramienta, ya que los usuarios deben aumentar su fuerza de agarre (Wimer et al., 2010).

\subsection{La norma ISO 10819:1996/2013 y la metodología para medir la eficacia de los guantes en} laboratorio

¿Cómo se mide la eficacia de un guante? En 1996, la Organización Internacional de Normalización (ISO) estableció la norma ISO 10819:1996 para probar y evaluar los guantes antivibratorios. Dicha norma describe un procedimiento en laboratorio con objeto de reproducir, de manera uniforme y fiable, la medida del efecto de aislación de los guantes. Esta norma fue revisada en el 2013 (ISO 10819:2013).

La norma ISO 10819 define criterios de aceptabilidad para que un guante sea reconocido como antivibratorio. Determina las condiciones y exigencias de medición en laboratorio, así como el análisis de los datos y el cálculo del factor de transmisión de las vibraciones de los guantes, en lo que se refiere a la transmisión de las vibraciones de un mango de una herramienta vibrante a la palma de la mano, en el rango de frecuencias comprendidas entre 31,5 Hertz (Hz) y $1250 \mathrm{~Hz}$.

El método recurre a una cantidad determinada de personas voluntarias ( $\mathrm{n}=3$ en 1996 y $\mathrm{n}=6$ en el 2013) que agarran un mango instrumentado unido a un sistema de excitación de vibración durante los ensayos en laboratorio. Se mantiene constante el nivel de aceleración. Los sujetos deben tomar el mango instrumentado manteniendo el antebrazo en dirección de las vibraciones, o sea según el eje z, el codo formando un ángulo de unos $90^{\circ}$. La medida de la aceleración se hace en la dirección del eje z, o sea a lo largo del antebrazo.

El mango, de $40 \mathrm{~mm}$ de diámetro, registra la aceleración y la fuerza de agarre se mantiene constante (30 Newtons - N). La fuerza de empuje se fija a $50 \mathrm{~N}$. Un pequeño adaptador ligero incorporado en un acelerómetro ubicado en la palma de la mano mide las vibraciones transmitidas al interior del guante. Al medir la amplitud de la vibración, simultáneamente en la superficie del mango y en la palma de la mano, se puede calcular la cantidad de vibración transmitida a la palma de la mano. La norma ISO define los 
factores de transmisión ponderada llamados TR. La ponderación de la aceleración queda establecida según la norma ISO 5349-1:2001.

21 Se determinan dos espectros en la norma ISO 10819:1996: un espectro medio (M) que cubre las frecuencias de $31,5 \mathrm{a} 200 \mathrm{~Hz}$ y un espectro de frecuencias altas (H) que cubre las frecuencias comprendidas entre 200 y $1250 \mathrm{~Hz}$. Cabe observar que la norma ISO 10819 descarta las bandas de tercios de octava inferiores a $31,5 \mathrm{~Hz}$, o sea las frecuencias de $6,3,8,10,12,5,16$ y $20 \mathrm{~Hz}$, las cuales son importantes en la evaluación del riesgo sobre la salud (Asaki \& Peterson, 2014).

Para que un guante sea reconocido como antivibratorio en el sentido de la norma ISO 10819:1996, debe cumplir con los siguientes criterios: un factor de transmisión (TR) en el espectro medio (M) de las frecuencias inferior a $1\left(\mathrm{TR}_{M}<1\right)$ y, en el espectro alto de las frecuencias $(\mathrm{H})$, un $\mathrm{TR}_{H}<0,6$. Esto significa que en el ámbito de las frecuencias medias, los guantes deben por lo menos no amplificar las vibraciones, mientras que en las más altas frecuencias, se necesita al menos una disminución de un 40 \% para el nivel de aceleración ponderada. Si se obtienen valores de TR superiores a 1, esto indica que el guante amplifica las vibraciones, mientras que los valores inferiores a 1 indican que el guante atenúa las vibraciones. Pese a estos criterios, los TR no indican la eficacia "real" del guante para atenuar la vibración ponderada en frecuencia y transmitida a la mano por las herramientas vibrantes. Por otro lado, la norma ISO 10819:1996 aclara que los dedos del guante deben tener las mismas propiedades (materiales y espesor) que la parte del guante que cubre la palma de la mano.

La norma ISO 10819:2013 refuerza los criterios de aceptabilidad para los guantes antivibratorios agregando un método de medición del espesor del material y definiendo los criterios para el espesor del guante en la palma de la mano y en los dedos. El espectro de las vibraciones comprende ahora las frecuencias entre 25 y $1250 \mathrm{~Hz}$. El criterio de aceptabilidad para las frecuencias medias pasa a $\mathrm{TR}_{\mathrm{M}}<0,9$ y se mantiene idéntico para el espectro $\mathrm{H}\left(\mathrm{TR}_{\mathrm{H}}<0,6\right)$. La norma menciona que el espesor del material antivibratorio puede ser disminuido a nivel de los dedos respecto del espesor en la palma de la mano (mínimo de 0,6 veces el espesor de la palma), basándose más en el confort que en los estudios de transmisibilidad a nivel de los dedos. Por otra parte, el espesor del guante en la palma de la mano no debería superar los $8 \mathrm{~mm}$.

\subsection{Principales críticas de la norma ISO 10819}

Desde la publicación de la norma en 1996, varios investigadores han examinado la metodología, han denunciado las limitaciones de la norma y han propuesto alternativas y mejorías que no han sido retenidas en la edición de la norma del 2013 (Paddan, 1996; Voss, 1996; Griffin, 1997; Hewitt, 1998; Dong et al., 2009; Welcome, Dong, Xu, Warren \& McDowell, 2012). Las principales críticas enunciadas y que siguen siendo de actualidad son las siguientes:

- Los espectros M y H actuales de la norma ISO 10819:1996 no representan la vibración producida por las numerosas herramientas vibrantes (Griffin, 1997; Dong, Rakheja, Schopper, Han \& Smutz, 2001).

- La idea de recurrir a los factores de ponderación de las frecuencias de la norma ISO 5349-1:2001 utilizados para estudiar la transmisibilidad de la vibración en la mano están siendo revisados. Es probable que una aceleración no ponderada pueda evaluar mejor la eficacia real de los guantes (Griffin, 1997). 
- La variabilidad de las medidas intra e interindividuales hace más difícil la distinción entre un guante antivibratorio y otro que no lo es. Además, la cantidad de sujetos es insuficiente para juzgar sobre la eficacia de diferentes tipos de guantes (Paddan \& Griffin, 1997; Griffin, 1998; Hewitt, 1998; Dong et al., 2001; Boileau, Rakheja \& Boutin, 2002; O’Boyle \& Griffin, 2001; Laszlo \& Griffin, 2011). Por otra parte, si la variabilidad entre los sujetos alcanza un $20 \%$, un guante podría ser reconocido como antivibratorio en un laboratorio y no en otro (Pinto, Stacchini, Bovenzi, Paddan \& Griffin, 2001; Dong et al., 2002/2004; Welcome, Dong, Xu, Warren \& McDowell, 2012).

- Una única postura durante las pruebas de laboratorio no es suficiente para representar las condiciones en que se opera sobre el terreno.

- El mango instrumentado puede tener una resonancia o un comportamiento dinámico indeseado en los intervalos de frecuencia medidos, lo que afecta la medida de la transmisibilidad (Paddan, 1996).

- La eficacia del guante se encuentra afectada por la fuerza de empuje y la posición de la mano y del brazo (Dong et al., 2004; Laszlo \& Griffin, 2011), pero, durante las pruebas, se estandariza una única combinación entre la fuerza de agarre de $30 \mathrm{~N}$ y la de empuje de $50 \mathrm{~N}$. Por lo cual, los resultados de laboratorio pueden ser significativamente diferentes de las medidas obtenidas sobre el terreno (Pinto, Stacchini, Bovenzi, Paddan \& Griffin, 2001; Shibata \& Maeda, 2008).

- Las mediciones del factor de transmisión de las vibraciones sólo se toman en la palma de la mano, sin tener en cuenta la transmisibilidad de las vibraciones a los dedos. En consecuencia, se debe desarrollar un modus operandi de medición a fin de medir el factor de transmisión de las vibraciones de los guantes en los dedos. Algunos estudios recientes lo mencionan. Sin embargo, la fijación del acelerómetro en los dedos afecta la fiabilidad de los resultados agregando una exigencia adicional al comportamiento biodinámico del sistema mano-brazo (Welcome, Dong, Xu, Warren \& McDowell, 2014/2014-a).

- La mayoría de los estudios analizaron la transmisibilidad de la vibración en la palma de la mano y en la misma dirección z que coincide con el eje de la fuerza de empuje (Hewitt, 1998, Dong et al.,, 2002/2004/2005/2009; Laszlo \& Griffin, 2011; Welcome et al., 2012). La norma postula que la reducción de la transmisibilidad medida en esa dirección del antebrazo es idéntica en todos los ejes. Sin embargo, McDowell, Dong, Welcome, Xu y Warren (2013) muestran que la transmisibilidad de la vibración es diferente en los tres ejes de medida mediante el ensayo de siete tipos de guantes. Los autores llegan a la conclusión que la medida de transmisibilidad de los guantes, conforme a la norma, sobreestima la atenuación total de los guantes. Los autores recomiendan medir todos los ejes y se cuestionan sobre los criterios de aceptabilidad de la norma.

- Los resultados de la evaluación de cuatro tipos de guantes en tres laboratorios distintos que recurrieron a la norma ISO 10819:1996 muestran que las diferencias experimentales son suficientemente importantes como para concluir en resultados contradictorios acerca de la capacidad de los guantes a cumplir con las exigencias definidas en la norma (Boileau \& Boutin, 2003).

\subsection{Factores que afectan la transmisibilidad de la vibración a la mano}

Para comprender adecuadamente en qué medida los guantes atenúan la transmisión de la vibración a la mano, hay que comprender los factores que entran en juego. 

de manera considerable por las fuerzas de acoplamiento de la mano (Pyykkö, Färkkilä, Toivanen, Korhonen \& Hyvärinen, 1976; Griffin, Macfarlane \& Norman, 1982; Aatola, 1989; Gurram, Rakheja \& Gouw, 1994/1995) y, en menor medida, por la amplitud de la aceleración (Reynolds \& Keith, 1977; Gurram, Rakheja \& Gouw, 1994/1995; Besa, Valero, Sufier \& Carballeira, 2007). De esta manera, es probable que estos factores influencien la transmisibilidad o la atenuación de los guantes (Griffin, 1990; Dong et al., 2004). fuerza de empuje que el operador aplica con los guantes (Gurram et al., 1994; Hewitt, 1998, Dong et al., 2002/2004; Smutz et al., 2002; Laszlo \& Griffin, 2011). Dong et al. (2002) mostraron que la fuerza de empuje tenía un efecto importante en las altas frecuencias, en un estudio que ponía a prueba dos tipos de guantes antivibratorios y fuerzas de empuje de $50 \mathrm{~N}$ y $100 \mathrm{~N}$, en sujetos expuestos a las vibraciones de martillos neumáticos. La transmisión de la vibración por el guante depende de la fuerza de empuje aplicada por la mano. El aumento de la fuerza aumenta la frecuencia de resonancia y aumenta la transmisibilidad de la vibración.

En el 2011, Laszlo y Griffin muestran que la transmisibilidad de la vibración por el guante depende completamente de la fuerza de empuje respecto de la amplitud de la vibración, sin tener en cuenta el material aislante de los guantes. Cabe notar que las fuerzas de empuje pueden alcanzar los $200 \mathrm{~N}$ en ciertas herramientas como los martillos neumáticos, las perforadoras o los taladros de asfalto (Dong et al., 2005). En este caso, resulta imposible concluir que un guante antivibratorio probado en laboratorio, con una fuerza de empuje de $50 \mathrm{~N}$, sea antivibratorio en dichas condiciones.

29 Parece ser que el comportamiento dinámico de los guantes es lineal, lo que implica que la transmisibilidad es independiente de la amplitud de la vibración. Hewitt (1998) mostró que las variaciones de amplitud de la vibración no afectan significativamente la transmisibilidad, con una diferencia mínima de $3 \%$ de un guante sometido a una vibración entre 3 y $20 \mathrm{~ms}^{-2}$. O'Boyle (2005) observa lo mismo, que al aumentar la amplitud de la vibración, la transmisibilidad de la vibración de tres tipos de guantes varía poco, salvo para los intervalos de frecuencias entre 160 y $400 \mathrm{~Hz}$.

\subsection{La eficacia de los guantes antivibratorios en laboratorio}

Cabe precisar de entrada que los estudios sobre la eficacia de los guantes se llevan a cabo en laboratorio y no sobre el terreno. Incluso en la actualidad, la eficacia real de estos guantes para atenuar la vibración no puede ser claramente demostrada. Hay que saber que debido al comportamiento biodinámico del sistema guante-mano, resulta difícil atenuar la vibración a bajas frecuencias. Sin embargo, cabe recordar que la mayoría de las herramientas vibrantes tienen una frecuencia dominante generalmente inferior a $250 \mathrm{~Hz}$. Por consiguiente, los guantes antivibratorios no podrían reducir significativamente la vibración de la mayor parte de las herramientas.

De manera general, los autores se cuestionan sobre la utilidad de los guantes antivibratorios con objeto de disminuir o atenuar la vibración transmitida a los dedos o a la mano (Rens et al., 1987; Griffin, 1990/1998; Padan \& Griffin, 2001; Dong et al., 2009; Welcome et al., 2012). La eficacia de atenuación o de aislación es débil y, en algunos casos, los guantes actúan como un transmisor de bajas frecuencias, como un 
amplificador de las frecuencias próximas a la frecuencia natural del sistema ofreciendo una atenuación mínima (alrededor de $300 \mathrm{~Hz}$ ) en las altas frecuencias, lo que corresponde, en este último caso, a una pulidora neumática que gira a 18000 vueltas por minuto (rpm) (Rens et al., 1987).

Desde el establecimiento de la norma ISO 10819, varios estudios han confirmado que la eficacia de los guantes varía según el espectro de frecuencia, el tipo de herramienta vibrante y la dirección del eje (Dong et al., 2002/2014; Rakheja et al., 2002; McDowell, Dong, Welcome, Xu \& Warren, 2013). La atenuación de las vibraciones es mejor en el eje z. Esto se debe especialmente al hecho de que la masa del sistema mano-brazo es más importante en esa dirección (McDowell et al., 2013).

Dong y otros (2004) demostraron que los guantes antivibratorios pueden reducir hasta un $15 \%$ la transmisión de la vibración en la palma de la mano, en frecuencias de más de $30 \mathrm{~Hz}$ y que el porcentaje de reducción puede aumentar cuando las frecuencias aumentan. Sin embargo, los guantes antivibratorios podrían amplificar la vibración en la palma de la mano en frecuencias de menos de $30 \mathrm{~Hz}$ y en los dedos en frecuencias de menos de $200 \mathrm{~Hz}$ (Dong et al., 2009).

En el 2017, Dong \& otros confirman que los guantes antivibratorios no reducen significativamente las vibraciones de las herramientas de baja frecuencia $(<25 \mathrm{~Hz})$ en la palma de la mano. Sin embargo, los guantes podrían reducir la vibración entre un 5 y 20 \% según la herramienta evaluada y la actividad del operador. Para alcanzar dicho resultado, los autores utilizan diferentes niveles medidos de aceleración ponderada de distintas herramientas vibrantes $\mathrm{y}$, con una fórmula matemática, calculan la reducción de la transmisibilidad de las vibraciones ofrecidas por diferentes tipos de guantes.

A raíz de problemas técnicos, los estudios sobre la transmisión de la vibración en los dedos son limitados (Griffin, Macfarlane \& Norman, 1982; Paddan \& Griffin, 2001). Nuevas tecnologías como el láser 3D facilitan la medición de la transmisión de la vibración en los dedos.

En general, los guantes tienen un efecto mínimo en la atenuación de la vibración transmitida a los dedos por varias herramientas. Sin embargo, ciertos tipos de guantes son más eficaces según el eje y la ubicación de la medida en el dedo. Los guantes alveolados y, en menor medida, los guantes de gel, amplifican la vibración en la punta de los dedos en el eje $\mathrm{x}$. Los guantes de neopreno son eficaces para reducir las vibraciones de alta frecuencia, pero amplifican las frecuencias inferiores a $100 \mathrm{~Hz}$ en la punta de los dedos (Welcome et al., 2014/2014-a).

Peterson, Asaki, Kudernatsch, Brammer y Cherniack (2014) muestran que la transmisibilidad de la vibración en los dedos, en nueve tipos de guantes analizados, es diferente de la de la palma de la mano, mientras que la transmisibilidad en el espectro $\mathrm{M}$, en la palma de la mano, muestra una atenuación según la norma y una amplificación en los dedos. Estos resultados sugieren que las medidas de transmisibilidad tomadas en la palma de la mano sólo pueden caracterizar incorrectamente la eficacia de un guante para atenuar la vibración y descuidar la exposición de toda la mano. Estos autores, contrariamente a otros, confirman que la medición en los dedos es posible y que debería ser considerada en otra norma. Welcome et al. (2014/2014-a) demostraron que la transmisibilidad de la vibración varía según las diferentes ubicaciones en cada dedo (Welcome et al., 2014/2014-a). Según estos autores, la eficacia de atenuación de los guantes en los dedos es inferior al $10 \%$, con una reducción media de menos del $5 \%$. 
Peterson, Asaki, Kudernatsch, Brammer \& Cherniack, 2014). De esta manera, el conocimiento de la transmisibilidad de las vibraciones en los dedos y su medición, gracias a las nuevas tecnologías, permitirán dar una opinión sobre la capacidad real de los guantes antivibratorios para atenuar las vibraciones transmitidas al sistema manobrazo.

\subsection{Modificaciones fisiológicas observadas en los humanos}

Disponemos de un solo estudio que expone la eficacia de los guantes antivibratorios a nivel vascular, en sujetos expuestos a las vibraciones de un mango instrumentado. El estudio de Mahbub et al. (2007) es un estudio experimental que mide el cambio del flujo sanguíneo y la temperatura en los dedos en sujetos normales que agarran un mango vibrante con o sin guantes. Los resultados muestran que el uso de los guantes aporta cambios en las frecuencias altas $(250 \mathrm{~Hz})$, pero ningún cambio en las bajas frecuencias $(31,5 \mathrm{~Hz})$.

40 Finalmente, un estudio de grupo (Jetzer, Haydon \& Reynolds, 2003) muestra que los trabajadores expuestos, a quienes se les propuso distintas posibilidades de prevención, como el uso de guantes antivibratorios, presentan menos síntomas relacionados con el síndrome de vibración. Sin embargo, resulta imposible aislar el efecto del uso de los guantes.

\subsection{Interferencia de los guantes en el desempeño laboral}

De manera general, los guantes de trabajo protegen a los trabajadores de las sustancias químicas, biológicas, de las cortaduras, abrasiones, quemaduras y vibraciones (Muralidhar, Bishu \& Hallbeck, 1999). Mantienen las manos calientes. También reducen la presión de contacto entre la mano y la herramienta (Muralidhar \& Bishu, 2000). Cuando el guante es más espeso, se aumenta el nivel de tolerancia a la presión ejercida en diferentes zonas de la mano. En general, los guantes pueden reducir la percepción táctil y la amplitud de los movimientos (Bellingar \& Slocum, 1993) y la fuerza de agarre (Chang \& Shih, 2007). Una reducción de la fuerza de agarre provoca un mayor esfuerzo de prensión para agarrar la herramienta y mantenerla en movimiento. Estos esfuerzos de prensión pueden incrementar el riesgo de trastornos musculoesqueléticos, como el síndrome del túnel carpiano (National Institute for Occupational Safety and Health, 1997).

Welcome et al. (2011) exponen, para diferentes tipos de guantes, una reducción de la fuerza de agarre así como ciertos obstáculos relacionados con el uso de estos guantes (Cuadro 1).

Cuadro 1: Reducción de la fuerza de agarre y obstáculos encontrados según el tipo de guantes

\begin{tabular}{|l|l|l|}
\hline $\begin{array}{l}\text { Tipo de } \\
\text { guantes }\end{array}$ & $\begin{array}{l}\text { Porcentaje (\%) de } \\
\text { reducción de la fuerza } \\
\text { de agarre }\end{array}$ & Obstáculos en el uso de los guantes \\
\hline
\end{tabular}




\begin{tabular}{|l|l|l|}
\hline Guante de gel & 42 & $\begin{array}{l}\text { Molesto, reducción de la destreza con los dedos, buen } \\
\text { aislante del frío, débil presión de contacto en la palma }\end{array}$ \\
\hline $\begin{array}{l}\text { Burbujas de } \\
\text { aire }\end{array}$ & 34 & $\begin{array}{l}\text { Distribución desigual de la presión de contacto debido a } \\
\text { la repartición de las burbujas de aire, pérdida de la } \\
\text { función antivibratoria a causa de la fuga de aire }\end{array}$ \\
\hline $\begin{array}{l}\text { Burbujas de } \\
\text { aire con la } \\
\text { bomba }\end{array}$ & 31 & $\begin{array}{l}\text { Exige un bombeo regular, pérdida de la función } \\
\text { antivibratoria }\end{array}$ \\
\hline Neopreno & 26 & Guante no antivibratorio, barato, confortable \\
\hline
\end{tabular}

Fuente: Dong et al. (2014)

Los guantes antivibratorios reducen la destreza manual (Christ, 1983; Rens et al., 1987; Pinto et al., 2001; Wimer et al., 2010). La pérdida de precisión puede engendrar un alto riesgo de accidente por operar de manera inadecuada las máquinas y herramientas (Kaulbars \& Walther, 2012). Los guantes pueden ser confortables (Wimer et al., 2010). Para ciertos autores, la incomodidad está relacionada con el aumento de la transpiración de la mano (Pinto et al., 2001); para otros autores, la incomodidad está asociada con la falta de flexibilidad del guante demasiado grueso (Kaulbars et al., 2012).

Cabeças y Milho (2009) mostraron que el uso de los guantes antivibratorios podían aumentar la fatiga muscular en el antebrazo (Dong \& otros, 2014). Las conclusiones de sus estudios coinciden con los estudios que muestran un aumento de la fatiga muscular en las manos con guantes respecto a las manos sin guantes, una disminución de la fuerza de agarre y ciertas modificaciones del electromiograma (Mital, Kuo \& Faard, 1994; Fleming, Jansen \& Hasson, 1997; Rock, Mikat \& Foster, 2001; Larivière et al., 2010).

Un estudio sobre la percepción del riesgo realizado con trabajadores, empleadores y médicos laborales mostró que estos últimos son capaces de identificar métodos de prevención, pero que se cuestionan sobre la utilidad y la eficacia real de los guantes para reducir la exposición (Tessier \& Turcot, 2013). Los participantes identificaron ciertos obstáculos en el uso de los guantes, como la falta de disponibilidad, de confort y los riesgos de seguridad. Rowley et al. (2011) van en el mismo sentido cuando mencionan determinadas barreras para el uso de los guantes, como la falta de disponibilidad sobre el terreno, la disminución del confort y la dificultad para realizar ciertas tareas con las manos enguantadas.

También hay que señalar que los trabajadores con el síndrome de vibración manobrazo y que presentan síntomas de entumecimiento, debilidad muscular y disminución de la fuerza de agarre corren el riesgo de que su situación se agrave con el uso de guantes antivibratorios.

\section{$3.9 ¿$ Existe una diferencia de eficacia entre los tipos de guantes?}

Es posible expresar su propia opinión acerca de la superioridad de un guante con respecto a otro, teniendo en cuenta la metodología de los estudios y los conocimientos actuales limitados sobre la transmisibilidad de la vibración a la palma de la mano y a los dedos. 
Debido a las exigencias de atenuación de las vibraciones relacionadas con los guantes antivibratorios, los organismos nacionales de investigación sobre la salud y la seguridad como el Health and Safety Executive (2005) no los recomiendan. Según la directiva europea Directiva 2002/44/CE del Parlamento europeo y del Consejo del 25 de junio de 2002, no se especifica el uso de los guantes antivibratorios en la jerarquía de medidas de prevención. Se propone más bien el uso de guantes para proteger a los trabajadores del frío y la humedad.

Según Kaulbars y Walther (2012), aunque sea actualmente imposible cuantificar el efecto protector de los guantes, se puede recomendar su utilidad en los siguientes lugares de trabajo:

- Con máquinas o herramientas que generan altas frecuencia superiores a los $150 \mathrm{~Hz}$, como una amoladora que gira a más de 900 rpm.

- Con herramientas que no requieren una fuerza de conducción precisa superior a $150 \mathrm{~Hz}$ o fuerzas de acoplamiento, como un compactador vibrante portátil.

- Con herramientas que requieren el uso de guantes para proteger del frío o de riesgos mecánicos.

Los lugares de trabajo en los que las vibraciones son transmitidas por el material o una pieza, es decir cuando se sostiene en las manos la pieza trabajada.

\section{ESTUDIO DE CAMPO}

51 Treinta (30) trabajadores participaron en la prueba de dos tipos de guantes antivibratorios provistos de la tecnología air gloves (guantes de cámara de aire) durante una semana para cada tipo de guante.

Según el fabricante, para los dos tipos de guantes, los resultados de los ensayos de laboratorio indican los siguientes factores de transmisión (TR): $\mathrm{TR}_{\mathrm{M}}=0,75 ; \mathrm{TR}_{\mathrm{H}}=0,45$ (ISO 10819:1996, Organisation internationale de normalisation, 1996). Sin embargo, el guante A, como se ha mencionado anteriormente, no es antivibratorio, ya que los dedos quedan al descubierto.

3 De los 30 participantes, 6 trabajadores probaron un solo par de guantes (Guante B). Se completaron y analizaron veintitrés (23) cuestionarios de evaluación (2. ${ }^{\circ}$ cuestionario). Dichos cuestionarios revelaron que 3 trabajadores presentaban la sintomatología asociada al síndrome de la vibración mano-brazo (síndrome del túnel carpiano en un trabajador y presencia de entumecimiento en los otros dos).

4 El cuadro 2 presenta las herramientas vibrantes utilizadas por los 30 trabajadores del estudio. Cada trabajador podía utilizar más de una herramienta en el marco de su trabajo, excepto los trabajadores forestales que sólo manipulan una sierra de cadena.

Cuadro 2: Herramientas vibrantes utilizadas por los trabajadores que participaron en el estudio

\begin{tabular}{|l|l|}
\hline Herramienta & Cantidad de trabajadores \\
\hline Arenadora orbital & 24 \\
\hline Amoladora & 16 \\
\hline
\end{tabular}




\begin{tabular}{|l|l|}
\hline Arenadora de banda & 8 \\
\hline Perforadora & 7 \\
\hline Bordeadora & 6 \\
\hline Pulidora & 2 \\
\hline Sierra de cadena & 2 \\
\hline Otra & 5 \\
\hline
\end{tabular}

El cuadro 3 presenta los tipos de guantes utilizados por los trabajadores del estudio antes del período de prueba. Cada trabajador podía usar más de un tipo de guante para realizar su trabajo.

Cuadro 3: Tipos de guantes utilizados durante el período de prueba

\begin{tabular}{|l|l|}
\hline Tipo de guante & Cantidad de trabajadores \\
\hline Algodón & 6 \\
\hline Antivibratorio & 4 \\
\hline Cuero & 3 \\
\hline Antideslizante & 2 \\
\hline Látex & 2 \\
\hline No mencionado & 2 \\
\hline Ninguno & 9 \\
\hline
\end{tabular}

\subsection{La evaluación de los guantes antivibratorios por los trabajadores}

El guante B, que cubre toda la mano, resulta muy caliente (18/23) y demasiado rígido (23/23) para los trabajadores. El abandono del estudio por parte de 6 trabajadores se explica por la rigidez del guante $\mathrm{B}$.

57 Se considera que los dos tipos de guantes son poco confortables, 15 trabajadores de 21 para el guante A y 20 de 23 para el guante B. La reducción del confort se debe al espesor del material antivibratorio en la palma de la mano y en los dedos. Los participantes tienen la sensación de que tienen las manos aprisionadas. La incomodidad de los guantes se explica por la falta de flexibilidad del material y el calor que se siente.

Los guantes dificultan el agarre de las herramientas. El espesor del guante en la palma de la mano impide que los dedos se junten, limitando el movimiento de pinza de los 
dedos. Como los guantes no se adhieren a la palma de la mano, esto produce en el trabajador una sensación de obstrucción.

La extensión del guante A a la altura de la muñeca, mediante el agregado de una banda de cuero, perjudica los movimientos de flexión y de extensión de la muñeca. Un trabajador que utilizaba regularmente una arenadora orbital se quejó de calambres en los músculos del antebrazo, debido a la mala postura causada por el guante A durante la manipulación de la herramienta.

Los trabajadores consideran que los guantes perjudican el desempeño de sus tareas (18 de 21 trabajadores para el guante A y 23 de 23 para el guante B) y provocan una reducción de la productividad (lentitud, retraso), ya que deben sacárselos repetidas veces para, por ejemplo, verificar la superficie del objeto pulido, tomar medidas, levantar pequeñas herramientas, atornillar, etc.

61 Los guantes representan también un riesgo para la seguridad. Un trabajador menciona que durante la manipulación de una sierra, debía ejercer una presión más fuerte para sostener la herramienta. Según este trabajador, la mano estaba cerca de la hoja, lo que podría haber sido peligroso si realizaba algún mal movimiento. Un trabajador forestal identificó la dificultad para accionar los comandos de la sierra de cadena, lo que podría causar accidentes.

De 21 participantes, 12 identifican un ajuste deficiente del guante A comparado a los 20 participantes de 22 para el guante B. Respecto de las críticas sobre el guante A, se exponen problemas de entumecimiento en los dedos, debido a que el guante que cubre la mitad del dedo se ajusta a la altura de la segunda falange.

Los trabajadores mencionan una pérdida de sensibilidad táctil y una pérdida de destreza manual con el uso de los guantes ya sea de tipo A o B. También explican que hay que realizar una mayor fuerza de agarre. Uno de los participantes confirma que el aumento de la fuerza de agarre implica una exigencia física suplementaria.

Para los participantes, los dos tipos de guantes aportan una cierta reducción de las vibraciones en la mano, pero las limitaciones asociadas a las tareas y el confort reducido de los guantes superan los beneficios de los guantes antivibratorios. Según los participantes, los guantes no causan enrojecimiento o ampollas en las manos. Varios trabajadores juzgaron pertinente usar los guantes para protegerse del frío, sobre todo en otoño e invierno, puesto que al manipular las herramientas, la misma herramienta y el mango se enfrían y las manos se congelan; es entonces cuando se aprecian los guantes. También se los aprecia porque protegen las manos del aceite de las herramientas y del polvo del pulido.

No se considera a los guantes como escurridizos cuando están sucios o mojados sea cual sea el modelo utilizado.

Entre las sugerencias para mejorar los guantes antivibratorios, la mayoría de los trabajadores identifica, en primer lugar, la necesidad de que sean más flexibles, pero también menos gruesos a la altura de la palma de la mano y que se ajusten mejor a la forma de la mano. Habría también que agrandar el guante a la altura de la muñeca para adaptarlo mejor a los movimientos de flexión y extensión de la mano. Habría que prever una aireación para atenuar el calor. Uno de los trabajadores sugiere mejorar la flexibilidad de los guantes en las articulaciones. 


\section{LIMITACIONES DEL ESTUDIO}

69 Cabe destacar que el presente estudio no trató acerca de la relación costo/eficacia de los guantes antivibratorios, pero hay que retener que la compra de estos guantes puede representar una fuente importante de inversión para los empleadores.

El único objetivo del estudio consistía en medir la adecuación del uso de los guantes con las herramientas vibrantes específicas. Los trabajadores que participaron en el estudio manipulaban diferentes herramientas vibrantes en su ámbito laboral.

71 Sería interesante hacer un estudio con trabajadores expuestos a vibraciones de alta frecuencia generadas por un solo tipo de herramienta, como las amoladoras, poniendo a su disposición diferentes tipos de guantes antivibratorios y analizando luego si el uso de los guantes resulta una medida eficaz en la reducción del riesgo del síndrome de vibración.

\section{CONCLUSIÓN}

El presente estudio tiene como objetivo documentar acerca de la eficacia de los guantes antivibratorios según los datos de la literatura y la aceptabilidad del uso de estos guantes por los trabajadores expuestos a las vibraciones de manos y brazos.

La revisión de la literatura revela que la eficacia de los guantes para atenuar las vibraciones es muy limitada. Como mucho, los guantes antivibratorios reducen entre un 5 y $20 \%$ las vibraciones de la palma de la mano, pero no aportan ninguna reducción de la transmisibilidad de la vibración en los dedos. Sin embargo, es justamente en los dedos que las vibraciones son más nocivas con el desarrollo del síndrome de la vibración. Además, no se puede demostrar la eficacia de los guantes antivibratorios en el medio laboral. Efectivamente, las condiciones de trabajo en las que se usan fuerzas de agarre y posturas variables son muy diferentes de las condiciones estandarizadas exigidas por la norma ISO 10819, por lo que resulta imposible asegurar el control de estos factores. En cuanto a los estudios de laboratorio, es difícil transponer los resultados para los trabajadores expuestos a una gama variada de herramientas cuyas frecuencias vibratorias varían, con dimensiones de mangos diferentes de las del mango instrumentado, una gran variedad de amplitud vibratoria y en contextos de trabajo variables.

74 La eficacia de los guantes antivibratorios depende de varios factores: las características biodinámicas del sistema mano-brazo (Dong et al., 2005), la variabilidad interindividual (Paddan \& Griffin 2001; Hewitt, 1998), la variabilidad intraindividual (O’Boyle \& Griffin, 2001; Hewitt, 1998), la mala alineación del adaptador (Dong, Rakheja \& Smutz, 2002; Smutz, 2001), entre otros. En consecuencia, aunque los guantes estén identificados como antivibratorios en el laboratorio, según las recomendaciones de la norma, resulta poco probable que aseguren la atenuación de las vibraciones en las manos. Como la atenuación de las vibraciones en frecuencias inferiores a los $300 \mathrm{~Hz}$ no es significativa y que la mayoría de las herramientas vibrantes se sitúan en esta gama de frecuencias, no se puede establecer, en cuanto al nivel de protección, si existe, que los guantes aportan dicha protección. El riesgo para la salud debido a la exposición a las vibraciones de manos y brazos permanece. Es necesario entonces realizar otros estudios a fin de 
evaluar mejor de qué manera atenuar la transmisibilidad de la vibración en manos y dedos.

En condiciones bien precisas, como la amoladura con amoladoras que producen altas frecuencias, Kaulbars y Walther (2012) recomiendan el uso de guantes antivibratorios. Sin embargo, las autoridades de la salud, como la HSE o la directiva europea, no hacen ninguna recomendación para ninguna herramienta vibrante. Se refieren más bien al uso de guantes para mantener las manos calientes evitando así los episodios de dedos blancos.

El estudio cualitativo realizado con trabajadores coincide con las conclusiones de ciertos autores en cuanto a los obstáculos identificados en relación con el uso de los guantes. Entre estos obstáculos, cabe señalar la disminución de la destreza manual (Wimer et al., 2010) y una incomodidad debido al calor y al sudor (Pinto et al., 2001), un aumento de la fuerza de agarre (Wimer et al., 2010), fatiga muscular (Rosponi, Lenzuni \& Fattorini, 2001; Cabeças \& Milho, 2009; Welcome et al., 2014/2014-a) y una disminución de la seguridad (Welcome et al., 2014/2014-a).

El uso de guantes puede, a lo sumo, ocasionar problemas de salud en los trabajadores, lo que no es despreciable. Además, la disminución de la fuerza de agarre y la pérdida de la sensibilidad táctil expuestas por los trabajadores que presentan el síndrome de vibración de manos y brazos agrava aún más los riesgos de la enfermedad debido al uso de los guantes antivibratorios. Sin embargo, sería necesario realizar otros estudios para validar esta hipótesis.

Por otra parte, el estudio permitió identificar otros obstáculos del uso de los guantes no presentados por la literatura inventariada, como la rigidez, la falta de flexibilidad en las articulaciones de los dedos, la interferencia en el desempeño y el rendimiento laboral, la aparición de efectos indeseables como el entumecimiento de los dedos y la dificultad para manipular las herramientas y accionar los comandos.

Además, los resultados cualitativos no permiten postular que la disponibilidad de los guantes antivibratorios en el medio laboral garantizaría su uso, contrariamente a lo que se expone en dos estudios (Rowley et al., 2011; Tessier \& Turcot, 2013).

Teniendo en cuenta los resultados de la revisión de la literatura y de los del estudio cualitativo en cuanto a la eficacia de los guantes y los obstáculos identificados, es preferible favorecer la puesta en práctica de medidas preventivas colectivas en los medios laborales. Entre estas medidas, cabe mencionar la compra de herramientas menos vibrantes, la reducción del tiempo de exposición, el mantenimiento preventivo de las herramientas, el respeto de las condiciones de uso de la herramienta recomendadas por el fabricante, el recurso a la fuerza de agarre o de empuje necesaria para sostener y guiar la herramienta y, por último, la disposición de los puestos de trabajo. 


\section{BIBLIOGRAFÍA}

Aatola, S. (1989). Transmission of vibration to the wrist and comparison of frequency response function estimators. Journal of Sound and Vibration, 131, 3, 497-507.

American National Standards Institute (ANSI) S3.40-2002/ISO 10819:1996. Mechanical vibration and shock - Hand-arm vibration - Method for the measurement and evaluation of the vibration transmissibility of gloves at the palm of the hand. Norme remplacée par ANSI S2.73-2014. Repéré à http:// infostore.saiglobal.com/store/details.aspx?ProductID=799800

Asaki, T., \& Peterson, D.R. (2014, June). Selecting tool-specific vibration-reducing gloves using ISO 5349 ANS ISO 10819 Measurements. Proceedings of the Fifth American Conference on Human Vibration. Ontario, Canada, 27-28. Repéré à Juin, 30, 2014, de http://www.uoguelph.ca/achv5/files/ ACHV\%205\%20Proceedings.pdf.

Besa, A.J., Valero, F.J., Sufier, J.L., \& Carballeira, J. (2007). Characterization of the mechanical impedance of the human hand-arm system: the influence of vibration direction, hand-arm posture and muscle tension. International Journal of Industrial Ergonomics, 37, 225-231.

Boileau, P-E., Rakheja, S., \& Boutin, J. (2002). Identification réduction de l'exposition aux vibrations main-bras chez les opérateurs d'outils portatifs vibrants (Rapport de recherche R-321). Montréal, Québec : Institut national de recherche Robert-Sauvé en santé et sécurité du travail (IRSST).

Boileau, P-E., \& Boutin, J. (2003). Évaluation interlaboratoire de gants destinés à réduire l'exposition aux vibrations main-bras (Rapport de recherche R-340). Montréal, Québec : Institut national de recherche Robert-Sauvé en santé et sécurité du travail (IRSST).

Cabeças, J.M., \& Milho, R.J. (2009). Anti-vibration gloves and the forearm efforts during tools Operations. Enterprise and Work Innovation Studies, 5, 59-67.

Chang, C.H., \& Shih, Y.C. (2007). The effects of glove thickness and work load on female hand performance and fatigue during an infrequent high-intensity gripping task. Applied Ergonomics, $38,3,317-324$.

Christ, E. (1983). Les gants de protection contre les vibrations: essais d'efficacité (Anti-vibration gloves: performance tests) (Cahiers de notes documentaires No. 110, $1^{\mathrm{er}}$ trimestre, 47-52. Institut national de recherche scientifique (INRS).

Directive 2002/44/CE du Parlement européen et du Conseil du 25 juin 2002. Exposition aux vibrations mécaniques. Repéré à

http://eur-lex.europa.eu/legal-content/FR/TXT/?uri=URISERV\%3Ac11145.

Dong, R.G., Rakheja, S., Schopper, A.W., Han, B., \& Smutz, W.P. (2001). Hand-transmitted vibration and biodynamic response of the human hand-arm: a critical review. Critical Reviews in Biomedical Engineering, 29, 4, 393-439.

Dong, R.G., Rakheja, S., Smutz, W.P., Schopper, A., Welcome, D., \& Wu, J.Z. (2002). Effectiveness of a new method (TEAT) to assess vibration transmissibility of gloves. International Journal of Industrial Ergonomics, 30, 1, 33-48.

Dong, R.G., Rakheja, S., \& Smutz, W.P. (2002). Evaluating anti-vibration performance of a glove using total effective transmissibility, International Journal of Industrial Ergonomics, 30, 33-48. 
Dong, R.G., McDowell, T.W., Welcome, D.E., Barkley, J., Warren, J., \& Washington, B. (2004). Effects of hand-tool coupling conditions on the isolation effectiveness of air bladder anti-vibration gloves. Journal of Low Frequency Noise, Vibration, and Active Control, 23, 4, 231-248.

Dong, R.G., McDowell, T.W., Welcome, D.E., \& Smutz, W.P. (2005). Correlations between biodynamic characteristics of human hand-arm system and the isolation effectiveness of antivibration gloves. International Journal of Industrial Ergonomics, 35, 205-216.

Dong, R.G., McDowell, T.W., Welcome, D.E., Warren, C., Wu, J.Z., \& Rakheja, S. (2009). Analysis of anti-vibration gloves mechanism and evaluation methods. Journal of Sound and Vibration, 321, 435-453.

Dong, R.G., Welcome, D.E., Peterson, D.R., Xu, S., McDowell, T.W., Warren, C. ..., \& Brammer, A. (2014, June). Tool-specific effectiveness of vibration-reducing gloves for attenuating palm-transmitted vibration. Proceedings of the Fifth American Conference on Human Vibration. Ontario, Canada, 29-39. Repéré à Juin, 30, 2014, de http://www.uoguelph.ca/achv5/files/

ACHV\%205\%20Proceedings.pdf

Fleming, S.L., Jansen, C.W., \& Hasson, S.M. (1997). Effect of work glove and type of muscle action on grip fatigue. Ergonomics, 40, 6, 601-612.

Goel, V.K., \& Rim, K. (1987). Role of gloves in reducing vibration: analysis for pneumatic chipping hammer. American Industrial Hygiene Association Journal, 48, 1, 9-14.

Griffin, M.J., Macfarlane, C.R., \& Norman, C.D. (1982). The transmission of vibration to the hand and the influence of gloves. In A.J. Brammer et W. Taylor W (Eds.), Vibration effects on the hand and arm in industry (pp. 103-116). New York: Wiley. Cité dans Dong, R.G., Rakheja, S. Smutz, W.P., Schopper, A., Welcome, D., \& Wu, J.Z. (2002). Effectiveness of a new method (TEAT) to assess vibration transmissibility of gloves. International Journal of Industrial Ergonomics, 30, 33-48.

Griffin, M.J. (1990). Handbook of human vibration. London: Academic Press.

Griffin, M.J. (1997). Measurement evaluation and assessment of occupational exposures to handtransmitted vibration. Occupational \& Environmental Medicine, 54, 73-89.

Griffin, M.J. (1998). Evaluating the effectiveness of gloves in reducing the hazards of handtranmitted vibration. Occupational \& Environmental Medicine, 55, 340-348.

Griffin M.J., \& Bovenzi, M. (2002). The diagnosis of disorders caused by hand-transmitted vibration: Southampton Workshop 2000. International Archives of Occupational and Envionmental Health, 75, 1-5.

Gurram, R., Rakheja, S., \& Gouw, G.J. (1994). Vibration transmission characteristics of the human hand-arm and gloves. International Journal of Industrial Ergonomics, 13, 217-234.

Gurram, R., Rakheja, S., \& Gouw, G.J. (1995). Mechanical impedance of the human hand-arm system subject to sinusoidal and stochastic excitations. International Journal of Industrial Ergonomics, 16, 135-145.

Health and Safety Executive. (1997). Vibrations solution. Practical ways to reduce the risk of hand-arm vibration injury. HSE book, hsg170. Repéré à http://www.hse.gov.uk/pubns/books/hsg170.htm.

Health and Safety Executive (2005). Hand-arm vibration. The control of vibration at work regulation 2005. Guidance on regulations. L140. Sudbury: HSE Books. ISBN 978007166125 1. Repéré à http:// www.hse.gov.uk/pubns/books/l140.htm. 
Hewitt, S. (1998). Assessing the performance of anti-vibration gloves a possible alternative to ISO 10819, 1996. The Annals of Occupational Hygiene, 42, 4, 245-252. Elsevier Science Repéré à http:// annhyg.oxfordjournals.org/content/42/4/245.full.pdf+html.

Jetzer, T., Haydon, P., \& Reynolds, D. (2003). Effective intervention with ergonomics, antivibration gloves, and medical surveillance to minimize hand-arm vibration hazards in the workplace. Journal of Occupational and Environmental Medicine, 45, 1312-1317.

Kaulbars, U., \& Walther, C. (JCHRV 2012, September). Certified anti-vibration gloves: Test methods and the limits to their effectiveness. The 20th Japan Conference on Human Response to Vibration, Osaka, Japan, Kinki University. Repéré à septembre, 30, de http://jchrv.net/documents/jchrv2012/4IFA_Kaulbars.pdf

Larivière, C., Tremblay, G., Nadeau, S., Harrabi, L., Dolez, P., Vu-Khanh, T., \& Lara, J. (2010). Do mechanical tests of glove stiffness provide relevant information relative to their effects on the musculoskeletal system? A comparison with surface electromyography and psychophysical methods. Applied Ergononomics, 41(2), 326-334. doi: 10.1016/j.apergo.2009.08.002

Laszlo, H.E., \& Griffin, M.J. (2011). The transmission of vibration through gloves: effects of push force, vibration magnitude and inter-subject variability. Ergonomics, 54, 5, 488-496. doi: $10.1080 / 00140139.2011 .562984$

Mahbub, H., Yokoyama, K., Laskar, S., Inoue, M., Takahashi, Y., Yamamoto, S., \& Harada, N. (2007). Assessing the influence of antivibration glove on digital vascular responses to acute hand-arm vibration. Journal of Occupational Health, 49, 165-171.

Marcotte, P. (2011). Prévention des vibrations. Communication présentée à Bruit et vibrations au travail, Paris, France. Repéré à Décembre, 16, de www.inrs.fr/inrs-pub/inrs01.nsf/ IntranetObject-accesParReference/HST_HST\%20223-4/\$File/HS\%20223-4.pd

McDowell, T.W., Dong, R.G., Welcome, D.E., Xu, X.S., \& Warren, C. (2013). Vibration-reducing gloves: transmissibility at the palm of the hand in three orthogonal direction. Ergonomics, 56, 12, 1823-1840. doi: 10.1080/00140139.2013.838642

Mital, A., Kuo, T., \& Faard, H.F. (1994). A quantitative evaluation of gloves used with non-powered hand tools in routine maintenance tasks. Ergonomics, 37, 2, 333-343.

Muralidhar, A., Bishu, R.R., \& Hallbeck, M.S. (1999). The development and evaluation of an ergonomic glove. Applied Ergonomics, 30, 6, 555-563.

Muralidhar, A., \& Bishu, R.R. (2000). Safety performance of gloves using the pressure tolerance of the hand. Ergonomics, 43, 5, 561-572. DOI: 10.1080/001401300184251

National Institute for Occupational Safety and Health (NIOSH) (1997). Musculoskeletal disorders and workplace factors. A critical review of epidemiologic evidence for work-related musculoskeletal disorders of the neck, upper extremity, and low back (Publication No. 97B141). DHHS (NIOSH). Repéré à http:// www.cdc.gov/niosh/docs/97-141/

O’Boyle, M.J., \& Griffin, M.J. (2001, June). Inter-subject variability in the measurement of the vibration transmissibility of gloves according to current standards. Proceedings of the 9 th International Conference on hand-arm vibration. Nancy, France: Institut National de Recherche et de Sécurité (INRS).

O’Boyle, M.J. (2005, September). Effect of vibration magnitude on glove material transmissability. Proceedings of UK 45th Conference on Human Response to Vibration. Liverpool, UK: Health and Safety Executive (HSE). 
Organisation internationale de normalisation (ISO) 10819:1996. Vibrations et chocs mécaniques -Vibrations main-bras -- Méthode pour mesurer et évaluer le facteur de transmission des vibrations par les gants à la paume de la main. http://www.iso.org/iso/fr/catalogue_detail.htm?csnumber=1418

Organisation internationale de normalisation (ISO) 10819:2013. Vibrations et chocs mécaniques -Vibrations main-bras -- Méthode pour mesurer et évaluer le facteur de transmission des vibrations par les gants à la paume de la main. http://www.iso.org/iso/fr/catalogue_detail.htm?csnumber=1418

Organisation internationale de normalisation (ISO) 5349-1:2001. Vibrations mécaniques -- Mesurage et évaluation de l'exposition des individus aux vibrations transmises par la main -- Partie 1: Exigences générales. http://www.iso.org/iso/fr/home/store/catalogue_tc/catalogue_detail.htm? csnumber $=32355$

Paddan, G.S. (1996, September). Effect of grip force and arm posture on the transmission of vibration through gloves. United Kingdom Informal Group Meeting on Human Response to Vibration, MIRA, Nuneaton.

Paddan, G.S., \& Griffin M.J. (1997). Individual variability in the transmission of vibration through gloves. In S.A. Robertson (ed.) Contemporary Ergonomics (p. 320-325). London: Taylor and Francis.

Paddan, G.S., \& Griffin, M.J. (2001). Use of seating to control exposures to whole-body vibration (Contract Research Report 335/2001). Health \& Safety Executive Books.

Peterson, D.R., Asaki, T., Kudernatsch, S., Brammer, A.J., \& Cherniack, M.G. (2014, June). Incorporating a finger adapter into iso 10819 assessments to measure the vibration transmissibility of gloves at the fingers. Proceedings of the 4th American Conference on Human Vibration ACHV 5. Guelph, Ontario: University of Guelph.

Pinto, I., Stacchini, N., Bovenzi, M., Paddan, G.S., \& Griffin, M.J. (2001, June). Protection Effectiveness of anti-vibration gloves: field evaluation and laboratory performance assessment. Vibration Injury Network. Communiqué présenté au 9th International Conference on Hand-Arm Vibration. Nancy, France.

Pyykkö, I., Färkkilä, M., Toivanen, J., Korhonen, O., \& Hyvärinen, J. (1976). Transmission of vibration in the hand-arm system with special reference to changes in compression force and acceleration. Scandinavian Journal of Work, Environment \& Health, 2, 2, 87-95.

Rakheja, S., Dong, R., Welcome, D., \& Schopper, A.W. (2002). Estimation of tool-specific isolation performance of antivibration gloves. International Journal of Industrial Ergonomics, 30, 71-87.

Rens, G., Dubrulle, P., \& Malchaire, J. (1987). Efficiency of conventional gloves against vibration. Ann Occup Hyg, 31, 2, 249-254.

Reynolds, D.D., \& Keith, R.H. (1977). Hand-arm vibration. Part I. Analytical model of the vibration response characteristics of the hand. Journal of Sound and Vibration, 51, 2, 237-253.

Reynolds, D.D., \& Jetzer, T. (1998, June). Use of air bladder technology to solve hand tool vibration problems. Proceedings of the $8^{\text {th }}$ International Conference on Hand-arm Vibration. Umeá, Sweden. Repéré à http://nile.lub.lu.se/arbarch/arb/1998/arb1998_14.pdf

Rock, K.M., Mikat, R.P., \& Foster, C. (2001). The effects of gloves on grip strength and three-point pinch. Journal of Hand Therapy, 14, 4, 286-290.

Rosponi, A., Lenzuni, P., \& Fattorini, L. (2001, Juin). Recrutement des unités motrices en fonction des vibrations transmises par les machines portatives. Notes scientifiques et techniques NS242. Actes du 9e Congrès international sur les vibrations mains-bras. Nancy, France. 
Rowley, K., Ajami, D., Gervais, D., Mooney, L., Solheim, A., Kudla, I., ..., \& Switzer-McIntyre, S. (2011). Glove use and education in workers with hand-arm vibration syndrome. Canadian acoustics, acoustique canadienne, 39, 2, 116-117.

Shibata, N., \& Maeda, S. (2008). Vibration-isolating performance of cotton work gloves based on newly issued JIS T8114. Industrial Health, 46, 477-483.

Smutz, W.P., Dong, R.G., Han, B., Schopper, A.W., Welcome, D.E., \& Kashon, M.L. (2002). A method for reducing adaptor misalignment when testing gloves using ISO 10819. The Annals, of Occupational Hygiene, 46, 3, 309-315.

Tessier, B., \& Turcot, A. (2012, Novembre). Perception à l'égard des risques reliés à l'utilisation des outils vibrants en milieu manufacturier et industriel. Agence de la santé et des services sociaux de la région de Chaudière-Appalaches.

Voos, P. (1996, July). Protection from hand-arm vibration by the use of gloves: possibility or fraud? Proceedings of Internoise (International Congress on Noise Control Engeneering, $\left.N^{\circ} 25\right)$. Liverpool, UK. Repéré à http://cat.inist.fr/?aModele=afficheN\&cpsidt=2724494.

Welcome, D.E., Dong, R.G., Xu, X.S., Warren, C., McDowell, T.W., \& Wu, J.Z. (2011). An investigation on the 3-D vibration transmissibility on the human hand-arm system using a 3-D scanning laser vibrometer. Canadian Acoustics, 39, 2, 44-45.

Welcome, D.E., Dong, R.G., Xu, X.S., Warren, C., \& McDowell, T.W. (2012). An evaluation of the proposed revision of the anti-vibration glove test method defined in ISO (1996). International Journal of Industrial Ergonomics, 42, 1, 143-155.

Welcome, D.E., Dong, R.G., Xu, S., Warren, C., McDowell, T.W., \& Wu, J. (2012-a, June). Effectiveness of anti-vibration gloves for reducing finger vibration. Proceedings of the Fourth American Conference on human vibration. Hartford, Connecticut.

Welcome, D.E., Dong, R.G., Xu, X.S., Warren, C., \& McDowell, T.W. (2014, June). Tool-specific performance of vibration-reducing gloves at the fingers. Proceedings of the Fifth American Conference on Human Vibration. Guelph, Ontario. http://www.uoguelph.ca/achv5/files/ ACHV\%205\%20Proceedings.pdf.

Welcome, D.E., Dong, R.G., Xu, X.S., Warren, C., \& McDowell, T.W. (2014-a). The effects of vibration-reducing gloves on finger vibration. International Journal of Industrial Ergonomics, 44, 1 , 45-59.

Wimer, B., McDowell, T.W., Xu, X.S., Welcome, D.E., Warren, C., \& Dong, R.G. (2010). Effects of gloves on the total grip strength applied to cylindrical handles. International Journal of Industrial Ergonomics, 40, 5, 574-583. NIOSHTIC No. 20037316. http://dx.doi.org/10.1016/j.ergon.2010.05.004.

\section{RESÚMENES}

El uso de guantes antivibratorios forma parte de las medidas de prevención fomentadas con el objeto de reducir la exposición a las vibraciones de manos y brazos. ¿Pero qué se sabe de la eficacia de estos guantes antivibratorios y de su aceptación por parte de los trabajadores? Se realizó una revisión de la literatura en Medline (PubMed) y EBSCOhost, en el período comprendido entre 1970 y 2014. Se hizo un estudio de campo con cuestionarios y entrevistas a 30 trabajadores a quienes se les pidió utilizar dos tipos de guantes antivibratorios durante un corto período para cada tipo de guante. 
Se completaron veintitrés (23) cuestionarios acerca del confort y de los obstáculos relacionados con el uso de guantes antivibratorios. Se analizaron doce (12) entrevistas individuales.

Varios trabajadores encontraron que los guantes propuestos eran incómodos y que dificultaban la realización del trabajo. Por otra parte, la revisión de la literatura revela que el grado de eficacia de estos guantes para disminuir las vibraciones es bajo.

No se ha demostrado la reducción de la exposición a las vibraciones de manos y brazos mediante el uso de guantes antivibratorios. Para los trabajadores, otros obstáculos más importantes están relacionados con el uso de estos guantes, como la disminución de la destreza manual, la incomodidad y una interferencia en el desempeño del trabajo.

O uso de luvas antivibráteis faz parte das medidas preventivas preconizadas para reduzir a exposição às vibrações mão-braço. Mas o que sabemos da eficácia daquelas luvas e da sua aceitabilidade pelos trabalhadores?

Uma análise da literatura foi realizada em Medline (PubMed) e EBSCOhost, para os anos de 1970 até 2014. Um estudo de terreno recorrendo a questionários e entrevistas foi desenvolvida com 30 trabalhadores que foram convidados a usar luvas antivibráteis durante um curto período para cada par de luvas.

Vinte e três (23) questionários relativos ao conforto e aos obstáculos do uso de luvas antivibráteis foram completados. Doze (12) entrevistas individuais foram analisadas.

Os dois tipos de luvas propostos foram considerados desconfortáveis e perturbadores a execução do trabalho para vários trabalhadores. A análise da literatura revela ainda que a eficácia das luvas na atenuação das vibrações é fraca.

A redução da exposição às vibrações mão-braço, pelo uso de luvas antivibráteis, não é demonstrada. Para os trabalhadores, os maiores obstáculos são relativos a uma diminuição da destreza, ao desconforto e a uma perturbação na execução do seu trabalho.

Le port de gants antivibratiles compte parmi les mesures préventives préconisées pour réduire l'exposition aux vibrations main-bras. Mais qu'en est-il de l'efficacité de ces gants antivibratiles et de leur acceptabilité pour les travailleurs?

Une revue de littérature a été menée dans Medline (PubMed) et EBSCOhost, pour les années 1970 à 2014. Une étude terrain avec questionnaires et entrevues a été réalisée auprès de 30 travailleurs qui ont été invités à porter deux types de gants antivibratiles sur une courte période pour chaque paire de gants.

Vingt-trois (23) questionnaires portant sur le confort et les obstacles au port de gants antivibratiles ont été complétés. Douze (12) entrevues individuelles ont été analysées. Les deux types de gants proposés ont été perçus comme inconfortables et gênaient l'exécution du travail pour plusieurs travailleurs. La revue de littérature révèle, par ailleurs, que l'efficacité des gants à atténuer les vibrations est faible.

La réduction de l'exposition aux vibrations main-bras, par le port de gants anti-vibratiles, n'est pas démontrée. Pour les travailleurs, des obstacles majeurs sont reliés à une diminution de la dextérité, de l'inconfort et une perturbation dans l'exécution de leur travail.

Wearing anti-vibration gloves is one of preventive measures recommended to reduce exposure to hand-arm vibration. But what do we know about the effectiveness of these anti-vibration gloves and about the workers' acceptance?

One literature review has been carried out in Medline (PubMed) and EBSCOhost, for the period from 1970 to 2014. A field study with questionnaires and interviews was done involving 30 workers that were invited to wear two types of anti-vibration gloves, for a short period of time each.

Twenty-three (23) questionnaires about comfort and drawbacks of wearing anti-vibration gloves were completed. Twelve (12) individual interviews were analysed. 
Both types of suggested gloves were considered uncomfortable and, according to several workers, they hindered the performance of the job. Furthermore, the literature review reveals that the effectiveness of the gloves to reduce vibrations is low.

Reducing hand-arm vibration exposure by wearing anti-vibration gloves has not been demonstrated. For the workers, the major problems are related with a decreasing dexterity, a discomfort and a disturbance at performing their jobs.

\section{ÍNDICE}

Keywords: hand-arm vibrations, anti-vibration gloves, exposure, personal protective equipment Mots-clés: vibrations main-bras, gants antivibratiles, exposition, équipement de protection individuelle

Palabras claves: vibraciones manos y brazos, guantes antivibratorios, exposición, equipamiento de protección individual

Palavras-chave: vibrações mão-braço, luvas antivibráteis, exposição, equipamento de protecção individual

\section{AUTORES}

\section{ALICE TURCOT}

Institut national de santé publique du Québec, 945, ave Wolfe, Québec, Canada, G1V 5B3

alice_turcot@ssss.gouv.qc.ca

\section{MICHEL LEHOUX}

Centre intégré de santé et des services sociaux de Chaudière-Appalaches, 1637, rue Notre-Dame Est, Thetford Mines, Québec, Canada, G6G 2V3

michel.lehoux@ssss.gouv.qc.ca 